\title{
Ontology-Based Data Access: A Survey
}

\author{
Guohui Xiao $^{1}$, Diego Calvanese ${ }^{1}$, Roman Kontchakov ${ }^{2}$, Domenico Lembo ${ }^{3}$, \\ Antonella Poggi ${ }^{3}$, Riccardo Rosati ${ }^{3}$ and Michael Zakharyaschev ${ }^{2}$ \\ ${ }^{1}$ KRDB Research Centre for Knowledge and Data, Free University of Bozen-Bolzano, Italy \\ ${ }^{2}$ Department of Computer Science and Information Systems, Birkbeck, University of London, UK \\ ${ }^{3}$ Dip. di Ing. Informatica Automatica e Gestionale, Sapienza Università di Roma, Italy
}

\begin{abstract}
We present the framework of ontology-based data access, a semantic paradigm for providing a convenient and user-friendly access to data repositories, which has been actively developed and studied in the past decade. Focusing on relational data sources, we discuss the main ingredients of ontology-based data access, key theoretical results, techniques, applications and future challenges.
\end{abstract}

\section{Introduction}

Ontology-based data access (OBDA, for short) is a semantic technology that has been developed since the mid 2000s [Poggi et al., 2008] with the aim of facilitating access to various types of data sources. It originates in real-world scenarios such as the one outlined below (see http://purl.org/slegge).

Example 1 Statoil (Equinor), a Norwegian multinational oil\&gas company, stores data in a large relational database (DB) Slegge with about 1500 tables (and 1700 views). Prior to making decisions on drilling wellbores, geologists at Statoil need to gather relevant information. For instance, geologists' information needs may include the following question:

(009) In my area of interest, return all pressure data tagged with key stratigraphy information with understandable QC attributes (and suitable for further filtering).

Translating such an information need into the standard database query language $S Q L$ is usually a big challenge for geologists, who are not supposed to know how Slegge is organised. In fact, the main table for wellbores has 38 columns; a four-table join with two additional filters is needed to obtain formation pressure for a wellbore, and stratigraphic information requires a join with 5 more tables. Using existing $S Q L$ templates and manipulating the answers is error prone, and calling an IT expert is time-consuming (it can take days or even weeks). OBDA offers a different approach to formalising and answering (009). Domain experts at Statoil designed a Subsurface Exploration ontology (SE) that captures terms of the user information needs such as Wellbore, hasFormationPressure, etc. IT experts wrote a mapping that declaratively connects (through $S Q L$ queries) the ontology predicates to the Slegge DB. The task of the geologist now is to reformulate (009) in the vocabulary of SE-possibly using a visual query interface such as OptiqueVQS ${ }^{1}$-as a query in the W3C standard SPARQL ${ }^{2}$, which could look as follows:

SELECT ?w ?depth ?strat_unit WHERE \{

?w a :Wellbore. ?w :hasMeasurement ?p .

?p a :Pressure. ?p :hasDepth ?depth

OPTIONAL \{ ?depth :inWellboreInterval ?strat_zone . ?strat_zone :hasUnit ?strat_unit \} \}.

This query retrieves all assignments to the variables ?w, ?depth, ?strat_unit in the SELECT clause that satisfy the conditions of the WHERE clause. The latter consists of triple patterns 'subject-predicate-object' (separated by dots) required to match the data. The first four triple patterns say that ?w is an instance of class Wellbore and has measurements, ?p, which are instances of Pressure and have their ?depth recorded by property hasDepth. The two triple patterns in OPTIONAL return additional information, if available, about the stratigraphic units ?strat_unit of the wellbore intervals for the depth measurements. It is optional in the sense that the variable ?strat_unit is assigned no value if the stratigraphic information is absent for the depth measurement.

An OBDA system would automatically rewrite this $S P A R Q L$ query using the ontology and mapping to a $S Q L$ query over the DB, optimise it, and evaluate it by Slegge.

In general, gathering information even from a company's DB is a hard task for non-IT-expert users. One of the main reasons is that DBs are usually designed to serve applications: their structure and meaning are obscure for most of the users; and the stored data is often redundant, mixed with information only needed to support company processes, and incomplete with respect to the business domain. Collecting, integrating, reconciling and efficiently extracting information from heterogeneous and autonomous data sources is regarded as a major challenge, with "most companies [...] capturing only a fraction of the potential value from data and analytics' ${ }^{3}$

The OBDA paradigm addresses this issue by providing ac-

\footnotetext{
${ }^{1} \mathrm{http}: / /$ optique-project.eu/training-programme/module-vqs

${ }^{2}$ http://www.w3.org/TR/sparql11-query

3“The age of analytics: competing in a data-driven world", McKinsey Global Institute, December 2016.
} 
cess to the data layer, consisting of autonomous data sources (e.g., DBs), through the mediation of a conceptual domain view, given in terms of an ontology, and the use of a declarative mapping between the data layer and the ontology. OBDA users do not have to know details of the data sources and can express their information needs as queries over the conceptual domain model. By applying knowledge representation and automated reasoning techniques, an OBDA system uses the ontology and mapping to reformulate the user queries into standard DB queries that are executed directly by the database management systems (DBMSs) of the sources. Thus, OBDA relies upon both KR\&R and DB technologies.

OBDA systems implementing this paradigm include Mastro [Calvanese et al., 2011], Morph [Priyatna et al., 2014], Ontop [Calvanese et al., 2017], Stardog 4 and Ultrawrap [Sequeda and Miranker, 2013]. They were adopted in many industrial projects and use cases, e.g., at Statoil and Siemens, the Italian Ministry of Economy and Finance [Antonioli et al., 2014], in projects on Smart Cities [López et al., 2015], Electronic Health Records [Rahimi et al., 2014], and Manufacturing [Petersen et al., 2017].

Over the past decade, the theory and practice of OBDA have become a hot topic in the areas of Knowledge Representation (Description Logics), Semantic Technologies and Databases, with numerous papers published in top CS journals (including AIJ, JACM, JAIR, TODS) and conferences, and deep connections with such prominent disciplines as Constraint Satisfaction and Circuit Complexity established.

In this brief survey, we introduce the framework of OBDA and discuss main results, techniques and challenges. We first describe the classical OBDA framework in Section 2. Then, in Section 3, we consider the process of query answering in OBDA. In Section 4, we focus on mapping management and analysis. In Section 5, we outline extensions of the classical OBDA framework. Finally, Section 6 discusses some of the most important research directions.

We assume the reader is familiar with the basics of databases (at a standard undergraduate DB course level).

\section{OBDA Framework}

We begin by presenting a formal framework for OBDA, distinguishing between the extensional (instance) and intensional (schema) levels. The former is given by a source $D B \mathcal{D}$ conforming to the data source schema $\mathcal{S}$ (which typically includes integrity constraints), and the latter by an $O B D A$ specification $\mathcal{P}=(\mathcal{O}, \mathcal{M}, \mathcal{S})$, where $\mathcal{O}$ is an ontology, $\mathcal{S}$ a data source schema and $\mathcal{M}$ a mapping from $\mathcal{S}$ to $\mathcal{O}$ (signatures of the ontology and schema are disjoint). The role of $\mathcal{O}$ is to provide the users with a high-level conceptual view of the data and a convenient vocabulary for their queries; it can also enrich incomplete data with background knowledge.

Example 2 The Subsurface Exploration ontology (SE) in Example 1 contains, among others, the following axioms, given in description logic (DL) syntax [Baader et al., 2017]:

\footnotetext{
${ }^{4}$ http://www.stardog.com

${ }^{5}$ http://optique-project.eu/results-downloads
}

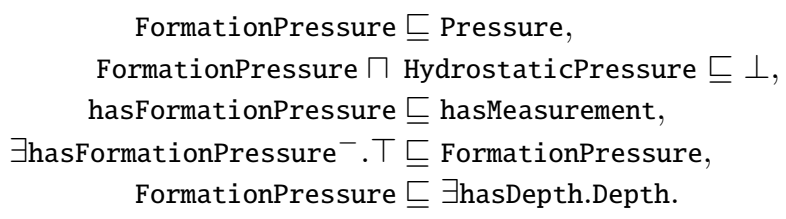

The first three are inclusions between, respectively, unary predicates (concepts in DL or classes in Semantic Web parlance) and binary predicates (roles or properties); their first-order (FO) equivalents look as follows:

$\forall x$ (FormationPressure $(x) \rightarrow$ Pressure $(x)$ ),

$\forall x$ (FormationPressure $(x) \wedge$ HydrostaticPressure $(x) \rightarrow \perp$ ),

$\forall x y$ (hasFormationPressure $(x, y) \rightarrow$ hasMeasurement $(x, y))$.

The fourth axiom restricts the range of hasFormationPressure, while the fifth involves existential quantification:

$\forall x y$ (hasFormationPressure $(y, x) \rightarrow$ FormationPressure $(x)$ ), $\forall x$ (FormationPressure $(x) \rightarrow \exists y(\operatorname{hasDepth}(x, y) \wedge \operatorname{Depth}(y)))$.

The mapping $\mathcal{M}$ in $\mathcal{P}$ specifies how the ontology predicates are populated by data from the source DB. In the SE example, each wellbore, which is identified by the column IDENTIFIER in the WELLBORE table, is given an IRI (Internationalised Resource Identifier) of the form http://slegger.gitlab.io/data\#Wellbore- $n$ to represent the wellbore in the ontology; in the sequel, we omit the prefixes and shorten such IRIs to Wellbore- $n$. Then the mapping connecting SE to the Slegge database contains the assertion

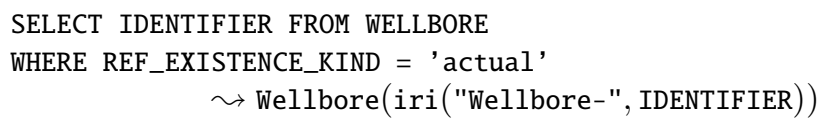

populating the class Wellbore with the answers to the $S Q L$ query to the left of $\leadsto$. In general, mapping assertions are of the form $\varphi(\boldsymbol{x}) \leadsto \psi(\boldsymbol{x})$, where $\varphi(\boldsymbol{x})$ and $\psi(\boldsymbol{x})$ are FOformulas in the signatures of $\mathcal{S}$ and $\mathcal{O}$, respectively. In our examples, we use $S Q L$ queries to conveniently represent the formulas $\varphi(\boldsymbol{x})$ (recall that WELLBORE has 38 columns). A special function iri (of variable arity) is used in $\psi(\boldsymbol{x})$ to construct IRIs for ontology objects: the parameters of iri are strings and DB columns (variables in $\boldsymbol{x}$ ), and the value of an iri term is the concatenation of its parameter values.

The pair $(\mathcal{P}, \mathcal{D})$ of an OBDA specification $\mathcal{P}$ and a source $\mathrm{DB} \mathcal{D}$ is called an $O B D A$ instance. To define its semantics, let $\mathcal{M}(\mathcal{D})$ be the minimal set of atoms in the signature of $\mathcal{O}$ that satisfies $\psi(\boldsymbol{a})$, for all $\varphi(\boldsymbol{x}) \leadsto \psi(\boldsymbol{x})$ in $\mathcal{M}$ and all tuples $\boldsymbol{a}$ of constants in $\mathcal{D}$ such that $\varphi(\boldsymbol{a})$ holds in $\mathcal{D}$. For example, in the SE setting, if the table WELLBORE contains

\begin{tabular}{rrr}
\hline IDENTIFIER & REF_EXISTENCE_KIND & $\ldots$ \\
\hline $16 / 1-29 \_S$ & actual & $\ldots$ \\
$30 / 8-5$ & actual & $\ldots$ \\
$33 / 10-12$ & planned & $\ldots$
\end{tabular}

then the mapping will produce the following two ground atoms (corresponding to ABox assertions or RDF triples):

Wellbore(Wellbore-16/1-29_S), Wellbore(Wellbore-30/8-5).

We call an FO-structure $\mathcal{I}$ over the signature of $\mathcal{O}$ a model of $(\mathcal{P}, \mathcal{D})$ and write $\mathcal{I} \models(\mathcal{P}, \mathcal{D})$, if $\mathcal{I} \models \mathcal{O}$ and $\mathcal{I} \models \mathcal{M}(\mathcal{D})$. Thus, the two ground atoms above form an FO-structure that 
is a model of our example OBDA instance. The additional mapping assertion

SELECT WELLBORE.IDENTIFIER, PRESSURE.PRESSURE_S

FROM WELLBORE, PRESSURE

WHERE WELLBORE.REF_EXISTENCE_KIND = 'actual' AND WELLBORE.WELLBORE_S = PRESSURE.FACILITY_S

$\leadsto$ hasFormationPressure(iri("Wellbore-", IDENTIFIER), iri("FP-", PRESSURE_S)),

which, for brevity, represents a join of three tables as a single 'table' PRESSURE, can produce the ABox assertion

hasFormationPressure(Wellbore-16/1-29_S, FP-1249).

The ontology will then imply the ground atoms

hasMeasurement(Wellbore-16/1-29_S, FP-1249), FormationPressure(FP-1249), Pressure(FP-1249),

which will hold in every model of our OBDA instance. Every model will also have to satisfy atoms hasDepth(FP-1249, a) and $\operatorname{Depth}(a)$, for some (possibly unknown) $a$.

The most important inference task in OBDA is query answering. Given a query $\boldsymbol{q}(\boldsymbol{x})$ in the signature of $\mathcal{O}$ with answer variables $\boldsymbol{x}$, a tuple $\boldsymbol{a}$ of constants in $\mathcal{D}$ is called a certain answer to $\boldsymbol{q}(\boldsymbol{x})$ over $(\mathcal{P}, \mathcal{D})$ if $\mathcal{I} \equiv \boldsymbol{q}(\boldsymbol{a})$, for every model $\mathcal{I}$ of $(\mathcal{P}, \mathcal{D})$. In our running example, FP-1249 is a certain answer to the conjunctive query (cf. Example 1)

$$
\exists x[\operatorname{Wellbore}(x) \wedge \text { hasMeasurement }(x, y) \wedge \operatorname{Pressure}(y)] \text {. }
$$

Conjunctive queries (CQs) are essentially SELECT-PROJECTJOIN $S Q L$ queries. We discuss the main inference task next.

\section{OBDA Query Answering}

To make query answering viable in practice, the OBDA paradigm relies on reducing the problem of finding certain answers to answering FO queries directly over the data. More precisely, given an OBDA specification $\mathcal{P}=(\mathcal{O}, \mathcal{M}, \mathcal{S})$, we say that a query $\boldsymbol{q}(\boldsymbol{x})$ is $F O$-rewritable if there is an FO query $\boldsymbol{q}^{\prime}(\boldsymbol{x})$ such that, for every source DB $\mathcal{D}$ for $\mathcal{P}$, a tuple $\boldsymbol{a}$ is a certain answer to $\boldsymbol{q}(\boldsymbol{x})$ over $(\mathcal{P}, \mathcal{D})$ iff $\mathcal{D} \models \boldsymbol{q}^{\prime}(\boldsymbol{a})$. The query $\boldsymbol{q}^{\prime}(\boldsymbol{x})$ is called an $F O$-rewriting of $\boldsymbol{q}(\boldsymbol{x})$ (with respect to $\mathcal{P}$ ). Thus, in the context of the example in Section 2, the following $S Q L$ query is an FO-rewriting of (1):

\section{SELECT "FP-" | PRESSURE_S FROM WELLBORE, PRESSURE \\ WHERE WELLBORE.REF_EXISTENCE_KIND = 'actual' AND WELLBORE.WELLBORE_S = PRESSURE.FACILITY_S,}

where || is the string concatenation operation. Since the FOrewriting of a query does not depend on the data, computing the certain answers to an FO-rewritable query has the same data complexity, viz. $\mathrm{AC}^{0}$, as classical $\mathrm{DB}$ query evaluation. Recall that, under data complexity, the data instance is the only input to the query answering problem, while the OBDA specification and query are fixed [Vardi, 1982].

The traditional approach [Poggi et al., 2008] to computing FO-rewritings of a query $\boldsymbol{q}(\boldsymbol{x})$ with respect to an OBDA specification $\mathcal{P}=(\mathcal{O}, \mathcal{M}, \mathcal{S})$ proceeds in two stages. In Stage 1 , the ontology-mediated query (OMQ) $(\mathcal{O}, \boldsymbol{q}(\boldsymbol{x}))$ is rewritten into an 'equivalent' FO-query $\boldsymbol{q}^{\prime}(\boldsymbol{x})$ in the signature of $\mathcal{O}$. In Stage $2, \boldsymbol{q}^{\prime}(\boldsymbol{x})$ is unfolded using the mapping $\mathcal{M}$ into an FO-query in the signature of $\mathcal{S}$, which gives the required FO-rewriting of $\boldsymbol{q}(\boldsymbol{x})$ with respect to $\mathcal{P}$. To ensure
FO-rewritability, the ontology and mapping languages need to be chosen with care.

\subsection{Ontology-Mediated Query Rewriting}

$O W L 2 Q L$ is a profile ${ }^{6}$ of the ontology language $O W L 2$, standardised by the $\mathrm{W} 3 \mathrm{C}$, that has been specifically designed for OBDA. It is based on the DL-Lite family [Calvanese et al., 2007; Artale et al., 2009], a suite of DLs closely related to conceptual modelling formalisms for DB design and software engineering. The DL-Lite constructs are illustrated by Example 2. OWL $2 Q L$ was tailored to ensure FO-rewritability of OMQs: if $\mathcal{O}$ is an OWL $2 Q L$ ontology, then any CQ $\boldsymbol{q}(\boldsymbol{x})$ is FO-rewritable with respect to the OBDA specification $\mathcal{P}_{\mathcal{O}}$ with ontology $\mathcal{O}$ and schema containing a unary table for each class and a binary table for each property, whose mapping is an isomorphism. An FO-rewriting of $\boldsymbol{q}(\boldsymbol{x})$ with respect to $\mathcal{P}_{\mathcal{O}}$ is called an $F O$-rewriting of the OMQ $(\mathcal{O}, \boldsymbol{q}(\boldsymbol{x}))$, which is also said to be $F O$-rewritable.

The first OMQ rewriting algorithm PerfectRef [Calvanese et al., 2007] was essentially based on backward chaining. To illustrate, consider the fragment $\mathcal{O}$ of the SE ontology in Example 2 and the CQ $\boldsymbol{q}(y)$ given by (1). PerfectRef rewrites OMQ $(\mathcal{O}, \boldsymbol{q}(y))$ into the union of the following CQs (UCQ):

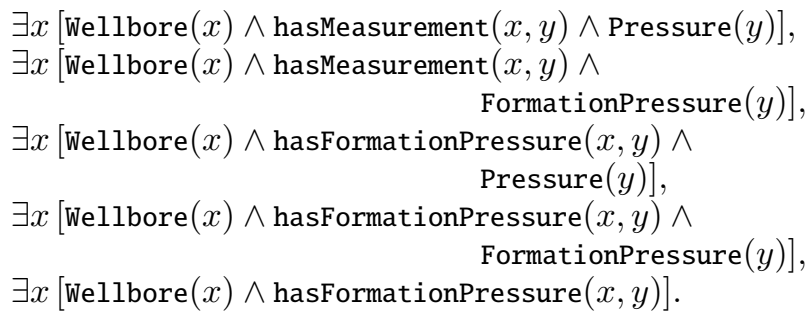

First experiments with Mastro [Calvanese et al., 2011] revealed that rewritings produced by PerfectRef were often prohibitively large for execution by DBMSs, which spurred the investigation of various OMQ rewriting techniques and optimisations. For example, optimisations based on CQ containment can significantly reduce the size of rewritings (the last CQ above subsumes the two preceding CQs); see, e.g., the work by Mora and Corcho [2013] and references therein for advances in this direction. Also, the UCQ above can be represented succinctly as a positive existential formula:

$$
\begin{aligned}
\exists x(\operatorname{Wellbore}(x, y) & \wedge[\text { hasFormationPressure }(x, y) \vee \\
\text { (hasMeasurement }(x, y) & \wedge \\
(\operatorname{Pressure}(y) & \vee \text { FormationPressure }(y)))]) .
\end{aligned}
$$

It turns out, however, that in the worst case, rewritings can be of exponential size even if represented more succinctly as positive existential formulas or non-recursive Datalog programs; rewritings in the form of arbitrary FO-formulas can be of superpolynomial size unless $\mathrm{NP} \subseteq \mathrm{P} /$ poly. For a comprehensive study of the succinctness problem and the combined complexity of OMQ answering (depending on the shape of CQs and the existential depth of ontologies), consult [Bienvenu et al., 2018], which also provides further references to various types of OMQ rewritings developed so far.

Ontology languages for which not all OMQs are uniformly FO-rewritable (because of LOGSPACE-hardness) were first

\footnotetext{
${ }^{6}$ http://www.w3.org/TR/owl2-profiles
} 
studied in 2006 [Calvanese et al., 2013], recasting also early results by Schaerf [1993] on DLs with CONP-hard query answering. Typical examples of non-FO-rewritable OMQs are

$$
\begin{aligned}
& (\{\forall x y(R(x, y) \wedge A(y) \rightarrow A(x))\}, \quad A(x)) \quad \text { and } \\
& (\{\forall x(A(x) \rightarrow B(x) \vee C(x))\}, \quad \exists \boldsymbol{x} \varphi(\boldsymbol{x})),
\end{aligned}
$$

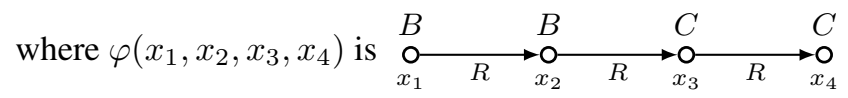

The former OMQ, expressible in the DL $\mathcal{E} \mathcal{L}$, encodes NLhard digraph reachability, while the latter, expressible in $\mathcal{A L U}$, is CoNP-complete [Gerasimova et al., 2017].

\subsection{Unfolding Rewritings with Mappings}

The FO-rewriting $\boldsymbol{q}^{\prime}(\boldsymbol{x})$ of an OMQ can be made executable over the source DB by means of unfolding. Consider first GAV (global-as-view) mappings, in which the assertions are of the form $\varphi(\boldsymbol{y}) \leadsto S(\boldsymbol{y})$ for a predicate $S$ (without iriterms). In this case, unfolding boils down to replacing each atom $S(\boldsymbol{z})$ in $\boldsymbol{q}^{\prime}(\boldsymbol{x})$ by the query $\varphi(\boldsymbol{z})$ from the mapping [Lenzerini, 2002] (which is similar to expanding a query with views in DBs or partial evaluation of Datalog programs).

The W3C standardised $R 2 R M L^{7}$ as a language for mapping relational DBs to RDF graphs (sets of RDF triples), where mapping assertions are of the form $\varphi(\boldsymbol{x}) \leadsto \psi(\boldsymbol{x})$, for a conjunction $\psi(\boldsymbol{x})$ of atoms over variables $\boldsymbol{x}$ and iriterms (expressed by means of IRI templates). Interestingly, De Giacomo et al. [2018] show that iri-terms can be used to encode more general GLAV mappings, where the righthand side query is a full-fledged CQ [Lenzerini, 2002]. This result heavily depends on the lack of functional properties and the unique name assumption (UNA) in $O W L 2$ QL [Calvanese et al., 2008]. In spite of this expressive power, all CQs are FO-rewritable with respect to OBDA specifications with OWL 2QL ontologies and R2RML mappings [Poggi et al., 2008]: indeed, any OMQ rewriting can be unfolded with an $R 2 R M L$ (or, equivalently, GLAV) mapping by using careful unification for query fragments [Calvanese et al., 2012].

Unfolding an FO-rewriting of an OMQ can result in an exponential blowup. For example, the class FormationPressure with the (simplified) mapping assertion

SELECT PRESSURE_S FROM PRESSURE

$\leadsto$ FormationPressure(iri("FP-", PRESSURE_S))

includes (as a subset) the range of hasFormationPressure; cf. Example 2. Thus, the rewriting of a CQ with an atom FormationPressure $(y)$ will have to 'expand' it both with itself and with hasFormationPressure $(z, y)$ for some $z$. Unfolding will then introduce a redundant $S Q L$ subquery for hasFormationPressure $(z, y)$. Indeed, this subquery gives no new answers compared to the subquery for FormationPressure $(y)$ because the latter subsumes the former, which selects only those tuples in PRESSURE that have matches in WELLBORE. The problem is exacerbated in the real SE because PRESSURE is a join of three tables. Kontchakov et al. [2014] and Sequeda et al. [2014] observed that optimisations removing such redundancies in rewritings of OMQs need to be made only once (as offline preprocessing) by com-

\footnotetext{
${ }^{7}$ http://www.w3.org/TR/r2rml
}

bining mapping and ontology. Indeed, the system can produce a mapping assertion for FormationPressure that gives all certain answers to FormationPressure $(y)$, so that its rewriting does not have to include predicates for redundant subqueries. Such mappings are called saturated or T-mappings.

Even with optimised saturated mappings, the $S Q L$ queries produced by unfolding may contain many redundant selfjoins and unions. Indeed, in the SE setting, data property name and object property hasDepth for the class FormationPressure are populated from columns IDENTIFIER and PRESSURE_S of the same table PRESSURE. Hence, the CQ

$$
\text { FormationPressure }(x) \wedge \operatorname{name}(x, y) \wedge \text { hasDepth }(x, z)
$$

is naturally unfolded into a join of 3 copies of table PRESSURE:

SELECT "FP-"||P1.PRESSURE_S, P2.IDENTIFIER

"PressureMeasuredDepth-" || P3.PRESSURE_S,

FROM PRESSURE P1, PRESSURE P2, PRESSURE P3

WHERE ("FP-"||P1.PRESSURE_S) = ("FP-" ||P2.PRESSURE_S)

AND ("FP-" || P1.PRESSURE_S) = ("FP-" || P3.PRESSURE_S).

Such redundancies can be detected and removed using integrity constraints of the DB schema (e.g., the primary key PRESSURE_S of PRESSURE above [Kontchakov et al., 2014]), or using additional constraints in the OBDA specification [Di Pinto et al., 2013; Hovland et al., 2016] (industrial DBs often contain few integrity constraints because they may impact system performance). We note that most of such semantic optimisations are well-known in DB theory; however, they are either not implemented in the state-of-the-art RDMSs (partly because naively unfolded $S Q L$ queries often contain obvious redundancies, such as the self-join above, not expected in human-written $S Q L$ queries) or use assumptions specific to OBDA (e.g., SPARQL joins are translated into joins of concatenated strings for the IRIs rather than standard database joins over columns as in the example above).

A promising direction in OBDA query optimisation is estimation of the evaluation cost for alternative equivalent forms of unfolded and rewritten queries in order to choose the best candidate; this can be done for OMQ answering [Bursztyn et al., 2015] or OBDA [Lanti et al., 2017].

\section{Mapping Management and Analysis}

Besides efficient query answering algorithms, design-time support is also crucial in OBDA, since the construction, debugging and maintenance of an OBDA specification are particularly demanding. Mapping creation and management is probably the most complicated OBDA design-time task, as the mapping specifies the semantics of the data sources in terms of the ontology, and so bridges the typically large conceptual gap between the source schema and the ontology. On the other hand, the form of the mapping and its interaction with the ontology affect query answering performance, and so should be taken into account by OMQ rewriting algorithms (cf., e.g., mapping saturation by Kontchakov et al. [2014]).

The first approach to formal analysis of OBDA mappings was proposed by Lembo et al. [2015], who focused on identifying inconsistencies and redundancies. A mapping $\mathcal{M}$ is called inconsistent with an ontology $\mathcal{O}$ if simultaneously activating all of its assertions leads to a contradiction in $\mathcal{O}$. 
Example 3 The axioms Well $\sqsubseteq$ Asset, Well $\sqsubseteq$ Facility, and Well $\sqcap \exists$ isoutsourcedTo. $\top \sqsubseteq \perp$ state that a well is both an asset and a company's facility, and it is not outsourced to an external operator. Consider the following mapping $\mathcal{M}$ :

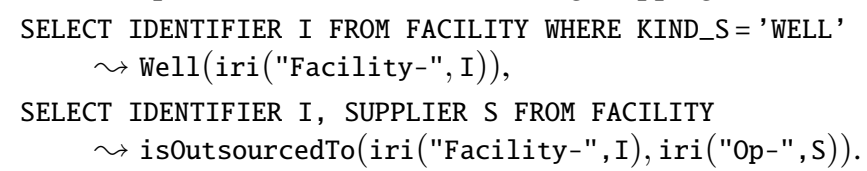

It is easy to see that $\mathcal{M}$ is inconsistent since every time the second assertion produces facts instantiating the ontology (i.e., it is activated), the first one is also activated, causing a violation of disjointness of Well and $\exists$ isOutsourcedTo. $\top . \quad \square$

A mapping $\mathcal{M}^{\prime}$ is redundant for an OBDA specification $\mathcal{P}=(\mathcal{O}, \mathcal{M}, \mathcal{S})$ if adding the assertions in $\mathcal{M}^{\prime}$ to $\mathcal{P}$ does not change its semantics, i.e., $(\mathcal{P}, \mathcal{D})$ and $\left(\left(\mathcal{O}, \mathcal{M} \cup \mathcal{M}^{\prime}, \mathcal{S}\right), \mathcal{D}\right)$ have the same models for every source DB $\mathcal{D}$. Besides the global notions of consistency and redundancy, Lembo et al. [2015] also consider their local counterparts, where the focus is on individual mapping assertions. They study the computational complexity of deciding both local and global consistency and redundancy for ontologies in OWL 2 and its tractable profiles, and different forms of mappings (GLAV or GAV). It turns out that mapping analysis can be carried out by composing standard reasoning tasks for the ontology and data sources, and is indeed not harder than these standard tasks.

The form of mapping analysis presented by Bienvenu and Rosati [2016] consists of a query-based notion of entailment and equivalence between OBDA specifications. In particular, two OBDA specifications are regarded as equivalent if they give the same answers to the same queries (in a certain class), for all possible source DBs. The paper studies the complexity of deciding entailment and equivalence between OBDA specifications with respect to different classes of queries (CQs or instance queries), ontology languages (of the DL-Lite family) and forms of mappings.

Lembo et al. [2017] focus on the evolution of OBDA specifications and consider the scenario where the ontology and/or the source schema change. This is a typical situation since, in applications, new data sources (or new portions of data sources) are usually incrementally added to an existing OBDA installation; moreover, the ontology often needs to be updated in the light of a deeper understanding of the domain of interest. In these cases, the mapping may have to be modified to restore consistency. Two notions of mapping repair are proposed. The first, called DM-Repair, considers as mapping repairs all maximal subsets of the original mapping that are consistent with the updated ontology and source schema. In Example 3, $\mathcal{M}$ is inconsistent with $\mathcal{O}$, and its repairs are the two mappings containing a single assertion each. The second notion, called EM-Repair, aims at maximising preservation of the information inferred by the original OBDA specification that is still consistent after the update. In Example 3, EM-Repairs are the DM-Repairs augmented with the following inferred mapping assertion:

SELECT IDENTIFIER I FROM FACILITY WHERE KIND_S='WELL' $\leadsto \operatorname{Asset}($ iri ("Facility-", I)).

Lembo et al. [2017] study the data and combined complexity of CQ entailment under the aforementioned notions of repair for $O W L 2 Q L$ ontologies and various forms of mappings.

We note that the works on mapping management discussed above do not make any assumptions on the way the mapping is originally designed. In order to support this activity, some approaches have recently been proposed, whose purpose is to automatically bootstrap the ontology and the mapping from a DB [Sequeda et al., 2011]. Typically, the bootstrapped ontology strongly depends on the abstraction level of the DB schema, which is rather low in real scenarios due to the fact that the database in practice plays the role of a (persistent) data structure for the enterprise applications. Consequently, the ontology needs to be manually refined to obtain a better conceptualisation of the domain of interest. BоотOX [Jiménez-Ruiz et al., 2015] provides some functionalities to help the designer improve the bootstrapped ontology by, e.g., aligning it to an existing domain ontology.

\section{Extensions}

We now survey extensions to the components of the OBDA framework that have been proposed in order to increase its expressive power and the scope of applications.

SPARQL, which has been adopted as the de facto standard query language in OBDA, was designed to deal with incomplete information (as exemplified by OPTIONAL in Example 1, where the variable ?strat_unit is not necessarily assigned a value). Its standard semantics is based on graph matching: the basic graph patterns (BGPs) of the query are matched to the data (which corresponds to CQ evaluation in classical DBs), and then the resulting sets of answers are combined using SPARQL operators such as OPTIONAL and UNION. Xiao et al. [2018b] develop an efficient translation of a large part of SPARQL into SQL, which uses column nullability in optimisations. In SPARQL 1.1, the entailment regimes were defined to account for reasoning in the ontological layer [Glimm and Krötzsch, 2010], a crucial aspect in OBDA. For example, the OWL 2 Direct Semantics entailment regime replaces graph matching for BGPs with entailment by a given OWL 2 ontology. This approach leads to a modular implementation of reasoning in existing $S P A R Q L$ engines. However, it also results in some counter-intuitive behaviour due to the lack of interaction between the certain answer semantics of BGPs and the OPTIONAL operator. Kostylev and Cuenca Grau [2015] and Ahmetaj et al. [2016] propose more intuitive semantics for the well-designed fragment of SPARQL.

Bag semantics. According to the $\mathrm{W} 3 \mathrm{C}$ specifications, every $R D F$ graph $\mathcal{M}(\mathcal{D})$ is a set of triples, but $S P A R Q L$ queries are evaluated under the bag semantics. However, the current OBDA research mostly adopts the set semantics for answering SPARQL queries. On the other hand, the bag semantics is important, in particular, for DB-style aggregate queries. Nikolaou et al. [2017] propose an alternative bag semantics for OBDA, where duplicate triples in $\mathcal{M}(\mathcal{D})$ are retained. They show that although such semantics makes answering CQs CONP-hard in general (and so not FO-rewritable), there is a large class of CQs rewritable to BALG, a generalisation of the relational algebra to bags.

Expressive Ontologies. The original OBDA paradigm relies on FO-rewritability of all OMQs with an OWL 2 QL ontol- 
ogy. There are different approaches to extending the expressive power of this DL-Lite-based ontology language. One was suggested by the DB community, which aimed at overcoming the restriction of DLs to unary and binary predicates only and designed various FO-rewritable fragments of the language of tuple-generating dependencies (aka Datalog ${ }^{ \pm}$or existential rules) - that is, the standard language for DB constraints [Krötzsch and Rudolph, 2011; Gottlob et al., 2014; König et al., 2015]. Another type of limitation, pointed out by the DL community, is that real-world ontologies often use constructs that are not available in DL-Lite, for example, $\exists R . C$ on the left-hand side of concept inclusions or $C \sqcup C^{\prime}$ on the right-hand side. Adding such constructs to DL-Lite would ruin the uniform FO-rewritablity of all OMQs; cf. Section 3.1. However, some useful OMQs with ontologies in an expressive language may still be FO-rewritable. A systematic investigation of the data complexity of answering individual OMQs in this non-uniform approach was launched by Lutz and Wolter [2017] and Bienvenu et al. [2014], who show, in particular, that FO- and Datalog-rewritability of expressive OMQs can be decided in exponential time. For recent results and further references, see the work by Hernich et al. [2017] and Lutz and Sabellek [2017]. The latter, for example, establish an $\mathrm{AC}^{0} / \mathrm{NL} / \mathrm{P}$ data complexity trichotomy for OMQs with $\mathcal{E} \mathcal{L}$ ontologies and atomic queries.

The approach of Botoeva et al. [2016] aims at extending OBDA to more expressive ontology languages while still leveraging the underlying relational technology for query answering. It does so by encoding part of the domain semantics of rich ontology languages in the mapping layer. More precisely, by replacing ontology axioms by additional mapping assertions, an OBDA specification with an expressive ontology is rewritten to an equivalent one with an OWL $2 Q L$ ontology, if possible, and approximated otherwise.

A more radical way of increasing expressiveness is to allow OMQ rewritings into query languages with polynomial (as opposed to $\mathrm{AC}^{0}$ ) data complexity, e.g., Datalog; a recent survey is provided by Bienvenu and Ortiz [2015].

Rules. An alternative approach to encoding more domain semantics in the ontology is to extend the OBDA paradigm with Datalog-style rules. For example, in the context of the SE ontology, the rule

$$
\begin{aligned}
& \text { intervalPerm }(i, v) \leftarrow \operatorname{extractedFrom}(c, i), \\
& \quad \text { hasCoreSample }(c, s), \operatorname{hasPerm}(s, p) \text {, valueInStdUnit }(p, v)
\end{aligned}
$$

says that permeability of a wellbore interval can be obtained by chaining four roles (binary predicates), while the two rules

$$
\begin{aligned}
& \text { ancestorUnit0f }(x, y) \leftarrow \operatorname{parentUnit0f}(x, y), \\
& \text { ancestorUnit0f }(x, y) \leftarrow \operatorname{parentUnit0f}(x, z), \\
& \text { ancestorUnit0f }(z, y)
\end{aligned}
$$

define ancestorUnitof by means of linear recursion on the binary relation parentUnitof. Xiao et al. [2014] extend the classical query rewriting algorithm for $S P A R Q L$ queries to deal with such rules by exploiting recursive common table expressions introduced in SQL:1999.
Spatial OBDA. The OGC standard GeoSPARQL query language $^{8}$ is an extension of SPARQL with geospatial features. It defines, in particular, a set of rules for transforming qualitative spatial queries into equivalent quantitative ones. For example, the qualitative $R D F$ triple $o_{1}$ geo: sfContains $O_{2}$ is true iff the geometry of object $o_{1}$ contains the geometry of $o_{2}$. So, given the GeoSPARQL query

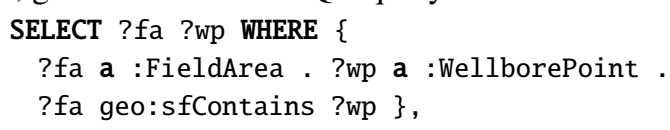

we obtain a SPARQL query with a union that contains, among others, the quantitative query that explicitly extracts geometries and relates them by the built-in function geo: contains:

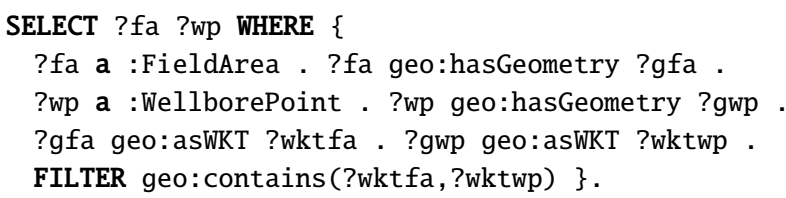

GeoSPARQL is supported by the approach proposed by Bereta and Koubarakis [2016], which relies on OBDA to access geospatial relational DBs (e.g., PostGIS, Oracle).

Temporal OBDA. Facilitating access to temporal (in particular, streaming) data has recently become a hot topic in the OBDA community. A typical example where temporal OBDA can be of great practical help is monitoring and analysing complex events based on timestamped sensor measurements stored in DBs. For instance, engineers at Siemens Remote Diagnostic Centres could be interested in active power trips of gas turbines, that is, events when the active power of a turbine was above $1.5 \mathrm{MW}$ for 10 seconds, and within 3 seconds after that there was a one-minute period when the active power was below $0.15 \mathrm{MW}$.

Two approaches to extending the classical OBDA languages with temporal constructs capable of capturing such events have been proposed. One approach suggested by Klarman and Meyer [2014], Borgwardt et al. [2015] and Kharlamov et al. [2017] is to retain OWL 2 QL as the ontology language under the assumption that the ontology axioms hold at all times, but enrich the query language with constructs from a standard temporal logic such as the linear temporal logic LTL, Halpern-Shoham's interval logic HS or the metric temporal logic $M T L$. For example, using $M T L$, one can encode the active power trip of a turbine $v$ as the query

$$
\begin{aligned}
& \boldsymbol{q}(v)=\operatorname{Turbine}(v) \wedge \square_{[0,60]}^{-} \mathrm{AP} \_B e l o w 0.15(v) \wedge \\
& \diamond_{[60,63]}^{-} \square_{[0,10]}^{-} \text {AP_Above 1.5 }(v) \text {, }
\end{aligned}
$$

where $\square_{\left[t_{1}, t_{2}\right]}^{-} A$ (respectively, $\diamond_{\left[t_{1}, t_{2}\right]}^{-} A$ ) holds at moment of time $t$ iff $A$ holds everywhere (respectively, somewhere) in the interval $\left[t-t_{2}, t-t_{1}\right]$. Although this approach usually preserves the data complexity and FO-rewritability of OMQs (see, e.g., [Baader et al., 2015]), it presupposes that users are capable of capturing complex events in temporal logic.

Alternatively and in the spirit of OBDA, one can shift the burden of representing complex temporal events to domain experts by adding temporal operators to ontology languages

\footnotetext{
${ }^{8}$ http://www.opengeospatial.org/standards/geosparql
} 
and keeping queries simple. For example, the ontology rule

$$
\begin{aligned}
& \text { AP_Trip }(v) \leftarrow \square_{[0,60]}^{-} \text {AP_Below0.15 }(v) \wedge \\
& \diamond_{[60,63]}^{-} \square_{[0,10]}^{-} \text {AP_Above1.5 }(v),
\end{aligned}
$$

which holds at all times, would reduce $\boldsymbol{q}(v)$ to a simple query Turbine $(v) \wedge$ AP_Trip $(v)$. Unfortunately, temporal operators often increase the complexity of OMQ answering and ruin FO-rewritability. Finding a trade-off between complexity and practically useful expressive power of temporal ontology languages remains a challenge; Artale et al. [2017] provide a recent survey. Brandt et al. [2017] and Kontchakov et al. [2016] demonstrate on a few real-world use cases sufficient expressiveness of non-recursive Datalog queries with MTL and HS rules such as (2) and reasonable scalability of their $S Q L$ rewritings (with window functions and aggregation).

Identifier Management for Data Integration. When the underlying data in OBDA is actually stored in a collection of DBs that need to be queried in an integrated way, we speak of ontology-based data integration. An important aspect that differentiates it from OBDA is the fact that the same conceptual entity (object) may be represented in different DBs by different identifiers. In order to express equivalence between entity identifiers, Calvanese et al. [2015] exploit the owl: sameAs construct in mappings. In addition, entities can be assigned canonical identifiers to avoid redundant query answers caused by owl: sameAs [Xiao et al., 2018a].

Materialisation and the Combined Approach. We described the classical approach to OBDA above, where no additional data is stored and used for query answering. This is also called virtual $O B D A$ because $\mathcal{M}(\mathcal{D})$ is virtual and not materialised. When the data may actually be extended, the DB engine can, for efficiency reasons, e.g., materialise some of the queries in the saturated mapping. Indeed, such materialised views can lead to drastically simpler $S Q L$ queries [Sequeda et al., 2014]. In the extreme, an OBDA system could attempt to materialise all logical consequences of the data, ontology and mapping, or, in DB parlance, to chase the data under the tuple-generating dependencies of the mapping and ontology [Abiteboul et al., 1995, Sections 8.4 and 10.2]. For most of the DL-based ontology languages, however, this is not possible as the chase is infinite. Still, even then the chase can be represented as a finite structure provided that queries are appropriately modified and/or their answers are filtered [Lutz et al., 2013].

\section{Perspectives}

We conclude by identifying a few important directions for future research, both in theory and practice.

Data Quality. The problem of assessing the overall quality of data requires measuring various data quality dimensions such as consistency (i.e., coherency with business rules), completeness (i.e., data contains the information needed for the task at hand) and currency (or freshness). When data comes from multiple independent data sources, OBDA provides a formal means to base data quality dimensions on a common ground, i.e., the domain ontology. Console and Lenzerini [2014] define a framework for data consistency in OBDA, considering coherence with the ontology axioms of both the content of the sources (extensional level) and their schema (intensional level). Algorithms and a complexity analysis are presented for checking different aspects of consistency for various classes of OBDA specifications. This investigation lays the foundations of a formal approach to data quality, focusing on the semantics of data sources. It remains to be broadened to other data quality dimensions beyond consistency.

Updates. With the exception of some preliminary efforts [De Giacomo et al., 2017], the OBDA framework has been mostly considered as read-only up to now. Obviously, the capability of the framework to react to insertion, removal or change of logically implied facts or axioms is a problem that deserves a systematic investigation.

Benchmarking. In order to assess the performance of OBDA query answering and understand the effectiveness of optimisation techniques, some benchmarks have been developed: e.g., [Lanti et al., 2015; Hovland et al., 2017] and the collection at obda-benchmark.org. Still, more challenging benchmarks, with complex ontologies, mappings and large data instances are needed, as well as benchmarks with tuneable components, which would allow one to study the impact of each of the components.

Non-Uniform OBDA. An ideal solution to the perennial expressiveness vs. complexity and rewritability problem would be an OBDA system that, for any query and OBDA specification given in expressive languages, could check the data complexity of query answering, identify a suitable type of rewriting and compute it. However, it still remains to be seen whether such algorithms are feasible in practice and whether simpler (syntactic) sufficient conditions of FOrewritability exist even for practically interesting classes of OMQs [Kaminski et al., 2016; Hansen and Lutz, 2017]. Another challenge is utilising mappings and DB integrity constraints, which restrict the class of possible data instances and thereby can drastically simplify rewritings.

Streaming Data. Recent years have witnessed a huge increase in the amount of streaming data that needs to be processed in real-time, also in connection to the growth of the Internet of Things. This affects data management technologies in general, but also Semantic Web technologies, which need to be extended so as to deal with streaming data efficiently; cf. the W3C RDF Stream Processing Community Group?. OBDA can provide the currently missing link between streams of raw (generally non-RDF) data and its highlevel view in terms of RDF triples, which would allow interoperability with the Semantic Web infrastructure. Calbimonte et al. [2010] describe an early proposal in this direction; Kharlamov et al. [2017] make first steps in developing analytics-aware temporal OBDA over streaming data.

Data Analytics. OBDA can be used to provide access to numerical data, representing, e.g., time, temperature or speed, using standard ontologies for these specific domains. Such structures are useful in formulating typical analytical tasks at a higher level of abstraction. This calls for extending the OBDA paradigm to the different types of numerical data, so as to support not only data access but also data analytics.

\footnotetext{
${ }^{9}$ http://www.w3.org/community/rsp
} 


\section{Acknowledgements}

We thank the reviewers for their suggestions. This work was supported by the OBATS project at the Free Univ. of BozenBolzano and by the Euregio (EGTC) IPN12 project KAOS.

\section{References}

[Abiteboul et al., 1995] S. Abiteboul, R. Hull, V. Vianu. Foundations of Databases. Addison Wesley, 1995.

[Ahmetaj et al., 2016] S. Ahmetaj, W. Fischl, M. Kröll, R. Pichler, M. Simkus, S. Skritek. The challenge of optional matching in SPARQL. In FoIKS, vol. 9616 of LNCS, 2016.

[Antonioli et al., 2014] N. Antonioli et al. Ontology-based data management for the Italian public debt. In FOIS, vol. 267 of FAIA, IOS Press, 2014.

[Artale et al., 2009] A. Artale, D. Calvanese, R. Kontchakov, M. Zakharyaschev. The DL-Lite family and relations. JAIR, 36, 2009.

[Artale et al., 2017] A. Artale, R. Kontchakov, A. Kovtunova, V. Ryzhikov, F. Wolter, M. Zakharyaschev. Ontology-mediated query answering over temporal data: A survey (invited talk). In TIME, vol. 90 of LIPICs, 2017.

[Baader et al., 2015] F. Baader, S. Borgwardt, M. Lippmann. Temporal query entailment in the description logic SHQ. J. Web Semantics, 33, 2015.

[Baader et al., 2017] F. Baader, I. Horrocks, C. Lutz, U. Sattler. An Introduction to Description Logic. CUP, 2017.

[Bereta and Koubarakis, 2016] K. Bereta, M. Koubarakis. Ontop of geospatial databases. In ISWC, vol. 9981 of LNCS, 2016.

[Bienvenu and Ortiz, 2015] M. Bienvenu, M. Ortiz. Ontology-mediated query answering with data-tractable description logics. In $R W$, vol. 9203 of LNCS, 2015.

[Bienvenu and Rosati, 2016] M. Bienvenu, R. Rosati. Query-based comparison of mappings in ontology-based data access. In $K R, 2016$.

[Bienvenu et al., 2014] M. Bienvenu, B. ten Cate, C. Lutz, F. Wolter. Ontology-based data access: A study through Disjunctive Datalog, CSP, and MMSNP. ACM TODS, 39(4), 2014.

[Bienvenu et al., 2018] M. Bienvenu, S. Kikot, R. Kontchakov, V. Podolskii, M. Zakharyaschev. Ontologymediated queries: Combined complexity and succinctness of rewritings via circuit complexity. JACM, 2018.

[Borgwardt et al., 2015] S. Borgwardt, M. Lippmann, V. Thost. Temporalizing rewritable query languages over knowledge bases. J. Web Semantics, 33, 2015.

[Botoeva et al., 2016] E. Botoeva, D. Calvanese, V. Santarelli, D. F. Savo, A. Solimando, G. Xiao. Beyond OWL 2 QL in OBDA: Rewritings and approximations. In AAAI, 2016.

[Brandt et al., 2017] S. Brandt, E. G. Kalayc1, R. Kontchakov, V. Ryzhikov, G. Xiao, M. Zakharyaschev. Ontology-based data access with a Horn fragment of Metric Temporal Logic. In $A A A I, 2017$.
[Bursztyn et al., 2015] D. Bursztyn, F. Goasdoué, I. Manolescu. Reformulation-based query answering in RDF: Alternatives and performance. PVLDB, 8(12), 2015.

[Calbimonte et al., 2010] J.-P. Calbimonte, Ó. Corcho, A. J. Gray. Enabling ontology-based access to streaming data sources. In ISWC, vol. 6496 of LNCS, 2010.

[Calvanese et al., 2007] D. Calvanese, G. De Giacomo, D. Lembo, M. Lenzerini, R. Rosati. Tractable reasoning and efficient query answering in description logics: The DL-Lite family. JAR, 39(3), 2007.

[Calvanese et al., 2008] D. Calvanese, G. De Giacomo, D. Lembo, M. Lenzerini, A. Poggi, R. Rosati, M. Ruzzi. Data integration through $D L$-Lite $_{\mathcal{A}}$ ontologies. In $S D K B$, vol. 4925 of LNCS, 2008.

[Calvanese et al., 2011] D. Calvanese et al. The Mastro system for ontology-based data access. Semantic Web, 2(1), 2011.

[Calvanese et al., 2012] D. Calvanese, G. De Giacomo, M. Lenzerini, M. Y. Vardi. Query processing under GLAV mappings for relational and graph databases. $P V L D B, 6$, 2012.

[Calvanese et al., 2013] D. Calvanese, G. De Giacomo, D. Lembo, M. Lenzerini, R. Rosati. Data complexity of query answering in description logics. AIJ, 195, 2013.

[Calvanese et al., 2015] D. Calvanese, M. Giese, D. Hovland, M. Rezk. Ontology-based integration of cross-linked datasets. In ISWC, vol. 9366 of LNCS, 2015.

[Calvanese et al., 2017] D. Calvanese et al. Ontop: Answering SPARQL queries over relational databases. Semantic Web, 8(3), 2017.

[Console and Lenzerini, 2014] M. Console, M. Lenzerini. Data quality in ontology-based data access: The case of consistency. In $A A A I, 2014$.

[De Giacomo et al., 2017] G. De Giacomo, D. Lembo, X. Oriol, D. F. Savo, E. Teniente. Practical update management in ontology-based data access. In ISWC, 2017.

[De Giacomo et al., 2018] G. De Giacomo et al. Using ontologies for semantic data integration. In A Comprehensive Guide through the Italian Database Research over the Last 25 Years, vol. 31 of Studies in Big Data. Springer, 2018.

[Di Pinto et al., 2013] F. Di Pinto et al. Optimizing query rewriting in ontology-based data access. In EDBT, 2013.

[Gerasimova et al., 2017] O. Gerasimova, S. Kikot, V. Podolskii, M. Zakharyaschev. On the data complexity of ontology-mediated queries with a covering axiom. In $D L$, vol. 1879 of CEUR, 2017.

[Glimm and Krötzsch, 2010] B. Glimm, M. Krötzsch. SPARQL beyond subgraph matching. In ISWC, 2010.

[Gottlob et al., 2014] G. Gottlob, G. Orsi, A. Pieris. Query rewriting and optimization for ontological databases. $A C M$ TODS, 39(3), 2014.

[Hansen and Lutz, 2017] P. Hansen, C. Lutz. Computing FO-rewritings in EL in practice: From atomic to conjunctive queries. In ISWC, vol. 10587 of LNCS, 2017. 
[Hernich et al., 2017] A. Hernich, C. Lutz, F. Papacchini, F. Wolter. Dichotomies in ontology-mediated querying with the guarded fragment. In PODS, 2017.

[Hovland et al., 2016] D. Hovland, D. Lanti, M. Rezk, G. Xiao. OBDA constraints for effective query answering. In RuleML, vol. 9718 of LNCS, 2016.

[Hovland et al., 2017] D. Hovland, R. Kontchakov, M. G. Skjæveland, A. Waaler, M. Zakharyaschev. Ontologybased data access to Slegge. In ISWC, vol. 10588 of $L N C S$, 2017.

[Jiménez-Ruiz et al., 2015] E. Jiménez-Ruiz et al. BootOX: Practical mapping of RDBs to OWL 2. In ISWC, vol. 9367 of LNCS, 2015.

[Kaminski et al., 2016] M. Kaminski, Y. Nenov, B. Cuenca Grau. Datalog rewritability of Disjunctive Datalog programs and non-Horn ontologies. AIJ, 236, 2016.

[Kharlamov et al., 2017] E. Kharlamov et al. Semantic access to streaming and static data at Siemens. J. Web Semantics, 44, 2017.

[Klarman and Meyer, 2014] S. Klarman, T. Meyer. Querying temporal databases via OWL 2 QL. In $R R$, vol. 8741 of LNCS, 2014.

[König et al., 2015] M. König, M. Leclère, M.-L. Mugnier, M. Thomazo. Sound, complete and minimal UCQrewriting for existential rules. Semantic Web, 6(5), 2015.

[Kontchakov et al., 2014] R. Kontchakov, M. Rezk, M. Rodriguez-Muro, G. Xiao, M. Zakharyaschev. Answering SPARQL queries over databases under OWL 2 QL entailment regime. In ISWC, vol. 8796 of LNCS, 2014.

[Kontchakov et al., 2016] R. Kontchakov, L. Pandolfo, L. Pulina, V. Ryzhikov, M. Zakharyaschev. Temporal and spatial OBDA with many-dimensional Halpern-Shoham logic. In IJCAI, 2016.

[Kostylev and Cuenca Grau, 2015] E. V. Kostylev, B. Cuenca Grau. Semantics of SPARQL under OWL 2 entailment regimes. In $D L$, vol. 1350 of CEUR, 2015.

[Krötzsch and Rudolph, 2011] M. Krötzsch, S. Rudolph. Extending decidable existential rules by joining acyclicity and guardedness. In IJCAI, 2011.

[Lanti et al., 2015] D. Lanti, M. Rezk, G. Xiao, D. Calvanese. The NPD benchmark: Reality check for OBDA systems. In EDBT, 2015.

[Lanti et al., 2017] D. Lanti, G. Xiao, D. Calvanese. Costdriven ontology-based data access. In $I S W C$, vol. 10587 of LNCS, 2017.

[Lembo et al., 2015] D. Lembo, J. Mora, R. Rosati, D.F. Savo, E. Thorstensen. Mapping analysis in ontologybased data access: Algorithms and complexity. In ISWC, vol. 9366 of LNCS, 2015.

[Lembo et al., 2017] D. Lembo, R. Rosati, V. Santarelli, D. F. Savo, E. Thorstensen. Mapping repair in ontologybased data access evolving systems. In IJCAI, 2017.

[Lenzerini, 2002] M. Lenzerini. Data integration: A theoretical perspective. In PODS, 2002.
[López et al., 2015] V. López, M. Stephenson, S. Kotoulas, P. Tommasi. Data access linking and integration with DALI: Building a safety net for an ocean of city data. In ISWC, vol. 9367 of LNCS, 2015.

[Lutz and Sabellek, 2017] C. Lutz, L. Sabellek. Ontologymediated querying with the description logic EL: Trichotomy and Linear Datalog rewritability. In IJCAI, 2017.

[Lutz and Wolter, 2017] C. Lutz, F. Wolter. The data complexity of description logic ontologies. LMCS, 13, 2017.

[Lutz et al., 2013] C. Lutz, I. Seylan, D. Toman, F. Wolter. The combined approach to OBDA: Taming role hierarchies using filters. In ISWC, vol. 8218 of LNCS, 2013.

[Mora and Corcho, 2013] J. Mora, O. Corcho. Engineering optimisations in query rewriting for OBDA. In $I$ SEMANTICS. ACM, 2013.

[Nikolaou et al., 2017] C. Nikolaou, E. Kostylev, G. Konstantinidis, M. Kaminski, B. Cuenca Grau, I. Horrocks. The bag semantics of ontology-based data access. In $I J$ CAI, 2017.

[Petersen et al., 2017] N. Petersen et al. Realizing an RDFbased information model for a manufacturing company A case study. In $I S W C$, vol. 10588 of $L N C S, 2017$.

[Poggi et al., 2008] A. Poggi, D. Lembo, D. Calvanese, G. De Giacomo, M. Lenzerini, R. Rosati. Linking data to ontologies. J. Data Semantics, 10, 2008.

[Priyatna et al., 2014] F. Priyatna, O. Corcho, J. F. Sequeda. Formalisation and experiences of R2RML-based SPARQL to SQL query translation using morph. In $W W W, 2014$.

[Rahimi et al., 2014] A. Rahimi, S.-T. Liaw, J. Taggart, P. Ray, H. Yu. Validating an ontology-based algorithm to identify patients with Type 2 Diabetes Mellitus in electronic health records. Int. J. Med. Inf., 83(10), 2014.

[Schaerf, 1993] A. Schaerf. On the complexity of the instance checking problem in concept languages with existential quantification. J. Intelligent Inf. Syst., 2, 1993.

[Sequeda and Miranker, 2013] J. F. Sequeda, D. P. Miranker. Ultrawrap: SPARQL execution on relational data. J. Web Semantics, 22, 2013.

[Sequeda et al., 2011] J. F. Sequeda, S. H. Tirmizi, O. Corcho, D. P. Miranker. Survey of directly mapping SQL databases to the Semantic Web. Knowl. Eng. Rev., 26, 2011.

[Sequeda et al., 2014] J.F. Sequeda, M. Arenas, D. P. Miranker. OBDA: Query rewriting or materialization? In practice, both! In ISWC, vol. 8796 of LNCS, 2014.

[Vardi, 1982] M. Vardi. The complexity of relational query languages (extended abstract). In STOC, 1982.

[Xiao et al., 2014] G. Xiao, M. Rezk, M. Rodriguez-Muro, D. Calvanese. Rules and ontology based data access. In $R R$, vol. 8741 of LNCS, 2014.

[Xiao et al., 2018a] G. Xiao et al. Efficient ontology-based data integration with canonical IRIs. In ESWC, 2018.

[Xiao et al., 2018b] G. Xiao, R. Kontchakov, B. Cogrel, D. Calvanese, E. Botoeva. Efficient Handling of SPARQL optional for OBDA. In ISWC, 2018. 